\title{
Optical simulations of advanced light management for liquid-phase crystallized silicon thin-film solar cells
}

\author{
Klaus Jäger, ${ }^{a, b}$ Grit Köppel, ${ }^{a}$ David Eisenhauer, ${ }^{a}$ Duote Chen, ${ }^{a, b}$ Martin Hammerschmidt,,${ }^{b} c$ \\ Sven Burger, ${ }^{b, c}$ and Christiane Becker ${ }^{a}$ \\ ${ }^{a}$ Helmholtz-Zentrum Berlin für Materialien und Energie GmbH, Kekuléstr. 5, D-12489 Berlin \\ ${ }^{b}$ Zuse Institute Berlin, Takustraße 7, D-14195 Berlin \\ ${ }^{c} \mathrm{JCM}$ wave GmbH, Bolivarallee 22, D-14050 Berlin
}

\begin{abstract}
Light management is a key issue for highly efficient liquid-phase crystallized silicon (LPC-Si) thin-film solar cells and can be achieved with periodic nanotextures. They are fabricated with nanoimprint lithography and situated between the glass superstrate and the silicon absorber. To combine excellent optical performance and LPC-Si material quality leading to open circuit voltages exceeding $640 \mathrm{mV}$, the nanotextures must be smooth.

Optical simulations of these solar cells can be performed with the finite element method (FEM). Accurately simulating the optics of such layer stacks requires not only to consider the nanotextured glass-silicon interface, but also to adequately account for the air-glass interface on top of this stack. When using rigorous Maxwell solvers like the finite element method (FEM), the air-glass interface has to be taken into account a posteriori, because the solar cells are prepared on thick glass superstrates, in which light is to be treated incoherently.

In this contribution we discuss two different incoherent a posteriori corrections, which we test for nanotextures between glass and silicon. A comparison with experimental data reveals that a first-order correction can predict the measured reflectivity of the samples much better than an often-applied zeroth-order correction.
\end{abstract}

\section{INTRODUCTION}

Liquid-phase crystallized silicon (LPC-Si) thin-film solar cells on glass are a promising novel approach with a current efficiency record of $13.2 \%{ }^{4,5}$ The LPC process is performed on a nanocrystalline Si layer on an interlayer stack on glass. Light management is used used to (i) reduce the reflective losses of the device via maximizing incoupling of light from the glass superstrate into the c-Si absorber, which has a high refractive index. Further, (ii) the absorption in the solar cell must be optimized via light trapping - especially at long wavelength. Here, light trapping is of special importance because the absorber in LPC-Si solar cells is only $5-20 \mu \mathrm{m}$ thick. Nanotextures for light management at the glass-Si interface can only be placed prior to the LPC process. Hence, they must be smooth in order to ensure LPC-Si with a high electronic quality. ${ }^{6}$

Figure 1 shows two types of periodic nanostructures between glass and LPC-Si, which we recently demonstrated: sinusoidal nanotextures ${ }^{1,7}$ and smooth anti-reflective three-dimensional textures (SMART). ${ }^{3}$ Both types are fabricated with nanoimprint lithography ${ }^{8,9}$ using high-temperature stable sol-gels, ${ }^{10}$ which are suited for the LPC process. LPC-Si solar cells on these textures have excellent material qualities and hence electric properties, such that open circuit voltages exceeding $615 \mathrm{mV}$ or even $640 \mathrm{mV}$ are measured for sinusoidal or SMART structures, respectively.

Devices using periodic nanotextures for light management can be simulated well with rigorous solvers of the Maxwell equations such as the finite element method (FEM). ${ }^{11}$ With FEM, the glass superstrate has to be taken into account a posteriori, because it is much too thick for a direct treatment with FEM. Domain composition methods ${ }^{12}$ which would allow to solve layer stacks with such thick layers using FEM, are not suited, because they treat the problem coherently, while sunlight in such a thick layer behaves incoherently.

Send correspondence to K.J.: E-mail: klaus .jaeger@helmholtz-berlin.de 
(a)

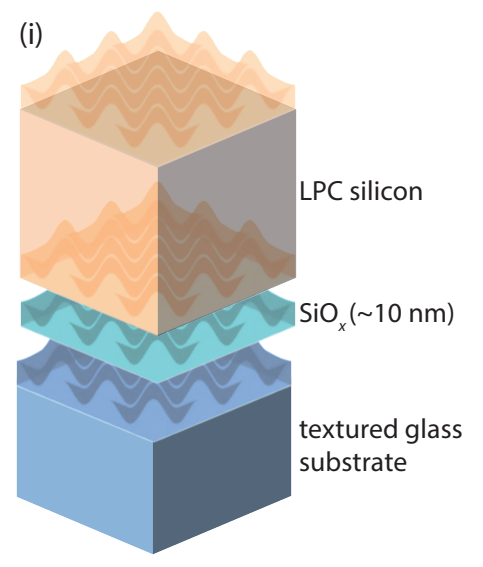

sinusoidal (ii)

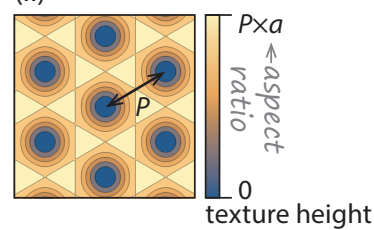

(iii)

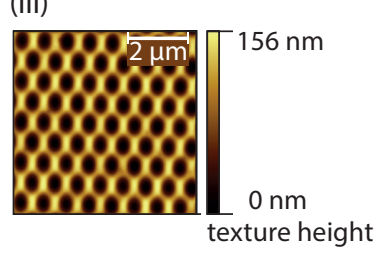

(b)

(i) (ii)

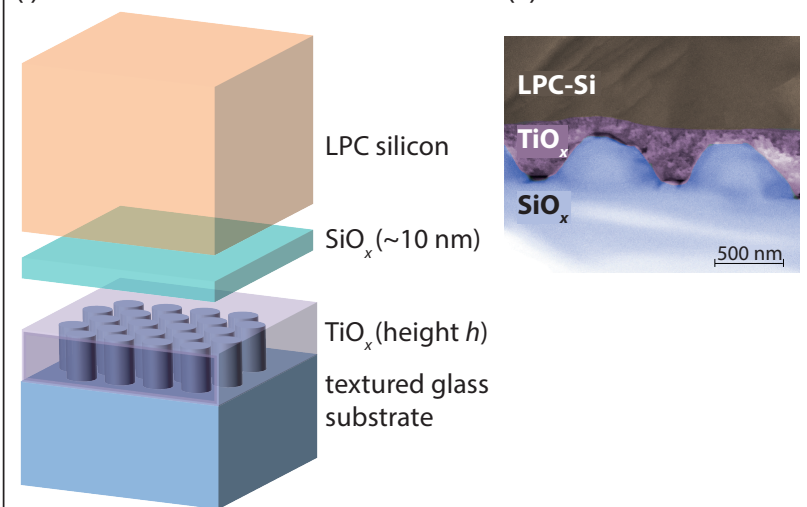

Figure 1. The two types of nanotextures for LPC-Si thin-film solar cells discussed in this paper:

(a) (i) The layer stack with a sinusoidal nanotexture, ${ }^{1}$ (ii) the sinusoidal texture used in the simulations, which is generated according to Eq. $(6)^{2}$ and (iii) an atomic force microscopy (AFM) measurement of a sinusoidally nanotextured sol-gel layer on glass with $750 \mathrm{~nm}$ pitch and about $150 \mathrm{~nm}$ texture height, hence an aspect ratio of about $a=20 \%$.

(b) (i) An illustration and (ii) a coloured SEM picture of smooth anti-reflective three-dimensional textures (SMART), ${ }^{3}$ which consist of silicon oxide $\left(\mathrm{SiO}_{x}\right)$ nanopillars in a hexagonal array covered by titanium oxide. SMARTs are characterized by the pitch $P$ [as in (a,ii)], the height $h$ and the filling fraction, given as the surface fraction covered by the $\mathrm{SiO}_{x}$ oxide nanopillars.

In this manuscript we describe a simple first-order correction to take the effect of the glass-silicon interface into account a posteriori. We compare the numerical results to experimental data and also to the zeroth-order correction, which only takes the initial reflection loss at the air-glass interface of about $4 \%$ into account.

This first-order correction can also be applied to other types of thin-film solar cells, when they have substrates in the range of millimeters such as perovskite solar cells ${ }^{13}$ or thin film silicon solar cells. ${ }^{14}$

\section{THEORY}

In numerical simulations, periodically structured thin-film layer stacks are usually treated with periodic boundary conditions on the side faces of the unit cell. Because of the perfect periodicity, the far-field reflection and transmission into the top and bottom infinite half spaces, which cover the layer stack, only happens into discrete and well-defined diffraction orders, as e.g. illustrated in Ref. 2 for hexagonal gratings. The reflectivity $R$ of the structure in the glass halfspace can be calculated with

$$
R(\lambda)=\frac{1}{\left|\mathbf{E}_{i}^{g}(\lambda)\right|^{2} \cos \theta_{i}^{g}(\lambda)} \sum_{j}\left|\mathbf{E}_{j}^{g}(\lambda)\right|^{2} \cos \theta_{j}^{g}(\lambda),
$$

where the electromagnetic field components $\mathbf{E}_{j}^{g}$ and the angles $\theta_{j}^{g}$ are output from the Maxwell solver. The subscript $i$ denotes the incident wave and the superscript $g$ denotes fields and angles in glass, as depicted in Fig. $2(\mathrm{a}) ; \lambda$ is the wavelength. The sum is taken over all channels into which the structure reflects.

The zeroth-order correction accounts only for the initial reflection of the air-glass interface. The reflectivity in air $R^{0}$ is calculated using

$$
R^{0}(\lambda)=R(\lambda)\left[1-R^{g}(\lambda)\right]+R^{g}(\lambda),
$$

where the superscript 0 denotes the zeroth-order correction and $R^{g}$ is the reflectivity of the air-glass interface (about $4 \%$ at normal incidence). ${ }^{2,15}$

The first-order correction takes into account that not all the light, which is reflected from the layer stack into the glass half space, is transmitted into air but that a part is reflected back into the layer stack by the glass-air 
(a) air

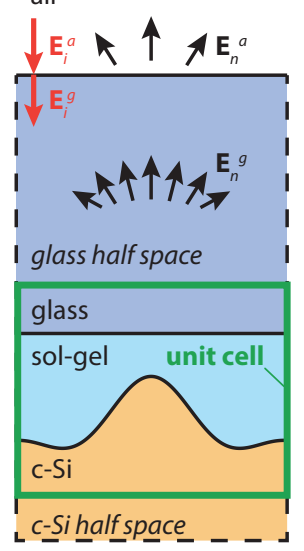

(b)

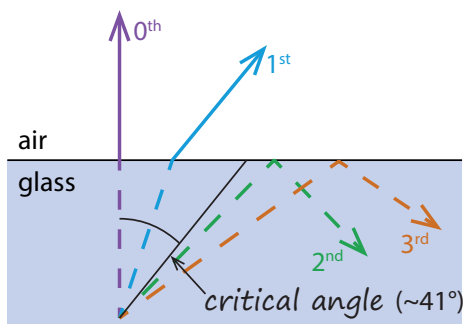

Figure 2. (a) Cross section through a periodic unit cell (enclosed by the green box) with a sinusoidal interface ${ }^{2}$ and the glass and silicon half spaces above and below the unit cell. The first-order correction described in this paper accounts for the interaction of the glass-air interface with light, which is reflected from the unit cell into the glass half space. The sketch depicts all the electric field components needed for this calculation. (b) Because of Snell's law the diffraction orders are present at much smaller angles in glass than in air. If a diffraction order is scattered into an angle larger than the critical angle, which is about $41^{\circ}$ for a glass-air interface, all the energy is directed back towards the layer stack.

interface. For its calculation, the electric field strengths $E_{n}^{a}$ and angles $\theta_{n}^{a}$ in air (superscript $a$ ) must be derived using the Fresnel equations and Snell's law, respectively,

$$
R^{1}=\frac{1}{\left[\left.\mathbf{E}_{i}^{a}\right|^{2} \cos \theta_{i}^{a}\right.} \sum_{j}[\underbrace{\left(t_{j}^{s} E_{s, j}^{g}\right)^{2}}_{E_{s, j}^{a}}+\underbrace{\left(t_{j}^{p} E_{p, j}^{g}\right)^{2}}_{E_{p, j}^{a}}] \cos \theta_{j}^{a}+R^{g} .
$$

Here, we have to decompose the $\mathbf{E}_{j}^{g}$ vectors into $s$ - and $p$-polarized components before multiplying them with the Fresnel coefficients $t_{n}^{s}$ and $t_{n}^{p}$, respectively. Note that $t_{j}^{s}$ and $t_{j}^{p}$ describe waves that are transmitted from glass into air. In contrast, $\mathbf{E}_{i}^{a}$ is connected to $\mathbf{E}_{i}^{g}$, which is used in Eq. (1), via transmission from air into glass.

For the decomposition, we first define a local orthonormal base $\left[\mathbf{n}_{k, j}, \mathbf{n}_{s, j}, \mathbf{n}_{p, j}\right]$,

$$
\begin{aligned}
\mathbf{n}_{k, j} & =\frac{\mathbf{k}_{j}}{\left|\mathbf{k}_{j}\right|}, \\
\mathbf{n}_{s, j} & =\frac{\mathbf{n}_{n} \times \mathbf{n}_{k, j}}{\left|\mathbf{n}_{n} \times \mathbf{n}_{k, j}\right|}, \\
\mathbf{n}_{p, j} & =\mathbf{n}_{k, j} \times \mathbf{n}_{s, j} .
\end{aligned}
$$

Here, $\mathbf{n}_{n}=(0,0,1)$ is the normal to the air-glass interface, $\mathbf{k}_{j}$ is the $j^{\text {th }} k$-vector of the electric field, which we obtain from the FEM simulation, and $\times$ denotes the vector product. If $\mathbf{n}_{n}$ and $\mathbf{n}_{k, j}$ are parallel, Eq. (4b) is replaced by

$$
\mathbf{n}_{s, j}=(1,0,0) .
$$

Now, $\mathbf{E}_{j}^{g}$ can be decomposed into $s$ - and $p$-polarized components via projecting $\mathbf{E}_{j}^{g}$ onto $\mathbf{n}_{s, j}$ and $\mathbf{n}_{p, j}$, respectively,

$$
E_{s, j}^{g}=\mathbf{E}_{j}^{g} \cdot \mathbf{n}_{s, j}, \quad E_{p, j}^{g}=\mathbf{E}_{j}^{g} \cdot \mathbf{n}_{p, j} .
$$

Note that the zeroth-order correction leads to an upper bound of the reflectivity, while the first-order correction leads to a lower bound. Higher-order corrections, where light that is reflected back into the layer again hits the nanotexture cannot be performed a posteriori - they require the calculation of the scattering matrix, which connects all incoming directions to all outgoing directions. The determination of the scattering matrix and the evaluation of higher scattering orders is out of the scope of this work. 
We illustrate the quantitative difference between the two corrections with a simple example: assume an optical system with $R=0.1$ under normal incidence, which only reflects specularly: with $R^{g}=0.04$ and using Eq. (2) we find $R^{0}=0.136$. The first-order correction takes the light into account that is reflected back into the layer stack at the air-glass interface, $R^{1}=R^{0}-R R^{g}\left(1-R^{g}\right)$, and hence $R^{1}-R^{0}=0.00384$, which probably might be neglected. But already if $R \gtrsim 0.3$, the absolute difference between the two corrections is about 0.01 , which is significant. Under oblique incidence or when the investigated system is strongly scattering, $R^{0}$ and $R^{1}$ may differ by tens of percent, as we will see below.

\section{SIMULATION AND EXPERIMENTAL DETAILS}

We compared the zeroth- and first-order corrections to each other and with experimental data for layer stacks consisting of an LPC-Si absorber on glass with a nanotexture in between, as illustrated in Fig. 1. As nanotextures we used sinusoidal ${ }^{1}$ and smooth anti-reflective three-dimensional textures (SMART). ${ }^{3}$

Sinusoidal nanotextures, illustrated in Fig. 1 (a), were already thoroughly studied numerically. ${ }^{2,16}$ For the simulations presented in this work, we use a negative cosine nanotexture, which is illustrated in Fig. 1(a,ii). Mathematically, it is - up to vertical and horizontal scaling - described with

$$
f(x, y)=-\cos (x) \cos \left[\frac{1}{2}(x+\sqrt{3} y)\right] \cos \left[\frac{1}{2}(x-\sqrt{3} y)\right] .
$$

We calculated the aspect ratio of the experimental sinusoidal samples by dividing the texture height, derived from AFM measurements, by the pitch. The error of the aspect ratio was deduced from the AFM error of $\pm 20 \mathrm{~nm}$. The reflectance spectra were obtained with an integrating sphere of $150 \mathrm{~nm}$ diameter, which is attached to a PerkinElmer LAMBDA 1050 spectrophotometer, at $\theta_{\text {in }}=8^{\circ}$ angle of incidence.

SMARTs, illustrated in Fig. 1 (b), consist of silicon oxide $\left(\mathrm{SiO}_{x}\right)$ nanopillars in a hexagonal array covered by titanium oxide $\left(\mathrm{TiO}_{x}\right)$. A thin $\mathrm{SiO}_{x}$ layer is used as antireflective coating.

The experimental samples have an LPC-Si layer thickness of about $10 \mu \mathrm{m}$, while in the simulations the silicon layer is considered to be infinitely thick. For wavelength shorter than about $600 \mathrm{~nm}$, all light that reaches the $10 \mu \mathrm{m}$-thick silicon layer will be absorbed. Hence, we compare simulated and measured reflectance spectra in a 350-600 nm range.

The reflected field components in glass $\mathbf{E}_{n}^{g}(\lambda)$ and the their angles $\theta_{n}^{g}(\lambda)$ with respect to the $z$-axis were obtained with the Maxwell solver JCMsuite, which utilizes the finite element method (FEM). ${ }^{17}$ The optical system studied for the simulations in this work is sketched in Fig. 2(a).

\section{RESULTS}

In glass, the different diffraction orders are present until much longer wavelength than in air, as illustrated in Fig. 2 (b). Figure 3 shows numerical and experimental $1-R$ spectra for a sinusoidally nanotextured sample with $750 \mathrm{~nm}$ pitch. Large differences are seen between the two corrections. The numerical results are shown for the angles of incidence of $\theta_{\text {in }}=0^{\circ}$ and $\theta_{\text {in }}=8^{\circ}$. Our spectrophotometer measures reflectance at $\theta_{\text {in }}=8^{\circ}$ angle of incidence. We observe that the $\theta_{\text {in }}=8^{\circ}$ curve matches much better with the measured data than the curve for normal incidence.

The difference between the spectra for $\theta_{\text {in }}=0^{\circ}$ and $8^{\circ}$ can be understood as follows: as mentioned above, perfectly hexagonal periodic unit cells scatter light into well-defined diffraction orders with six channels each (except the zeroth). ${ }^{2}$ At normal incidence, the spectra show sharp edges, which refer to the threshold wavelengths at which a certain diffraction order cannot leave the structure any more. At $\theta_{\text {in }}=8^{\circ}$, the six channels of one diffraction order are scattered into different angles and hence vanish at different threshold wavelengths. Hence, instead of a sharp edge several less pronounced edges are observed, as seen in Fig. 3.

Figure 4 shows results for a SMART texture, as illustrated in Fig. 1 (b). These results were obtained for two different filling fractions, a structure height of $h=50 \mathrm{~nm}$ and $750 \mathrm{~nm}$ pitch. As for the sinusoidal textures, the first-order correction leads to significantly smaller reflectivity as the zeroth-order correction. However, the difference is smaller than in the sinusoidal case [Fig. 3], probably because the layer stack containing $\mathrm{TiO}_{x}$ already has an certain antireflective effect. Also experimental data is shown; its filling fraction is about $50 \%$. However, 


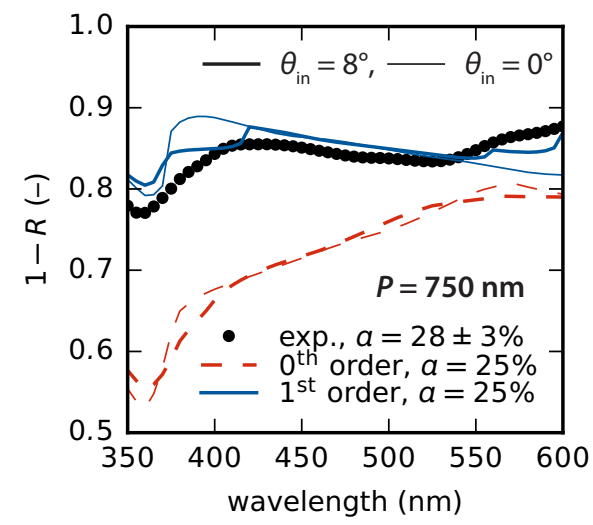

Figure 3. Numerical and experimental $1-R$ spectra for the sinusoidally textured layer stacks illustrated in Fig. 1 (a). The shown data is for $750 \mathrm{~nm}$ pitch. Numerical results were calculated with the $0^{\text {th }}$ - and $1^{\text {st }}$-order corrections, as defined in Eqs. (2) and (3), respectively. The two corrections differ because not all diffraction orders that are present in glass can propagate into air [see Fig. 2 (b)]. Simulation results are shown for two angles of incidence: $\theta_{\text {in }}=0^{\circ}$ (thin lines) and $\theta_{\text {in }}=8^{\circ}$ (thick lines). Experimental results were obtained with $\theta_{\text {in }}=8^{\circ}$.

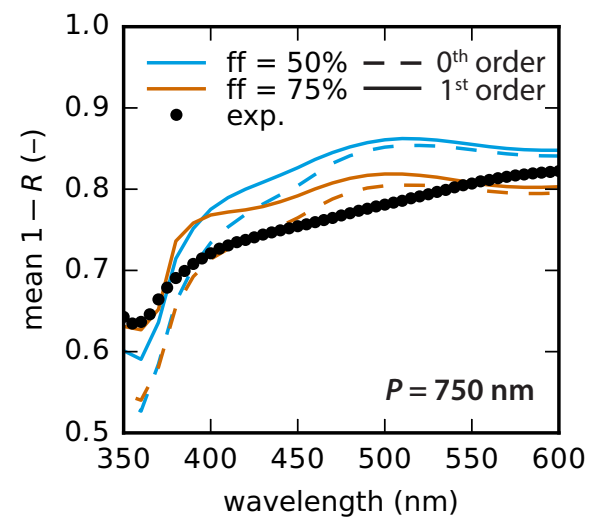

Figure 4. $1-R$ spectra for the SMART layer stacks illustrated in Fig. 1 (b) with two different filling fractions and $\approx$ $50 \mathrm{~nm}$ SMART height for $750 \mathrm{~nm}$ pitch. Numerical results were calculated with the $0^{\text {th }}$ - and $1^{\text {st }}$-order corrections, as defined in Eqs. (2) and (3), respectively. The two corrections differ because not all diffraction orders that are present in glass can propagate into air [see Fig. 2 (b)]. Experimental data is shown for a filling fraction of around 50\%. Simulation results are shown for $\theta_{\text {in }}=0^{\circ}$ angle of incidence; experimental results were obtained with $\theta_{\text {in }}=8^{\circ}$. 
the simulation curve for $75 \%$ filling fraction matches better with the experimental data. The simulations can reproduce the trend seen experimentally, especially the peak in reflectivity at $370 \mathrm{~nm}$ wavelength is reproduced. The differences probably are caused by differences between the experimental and the simulated layer stack. As seen in the SEM picture in Fig. 1 (b,ii), the interface between the SMART and silicon is not flat, in contrast to what is assumed in the simulations. Further, the pillars are rounded at the top and not perfect cylinders.

\section{SUMMARY AND OUTLOOK}

When simulating the optics of periodically nanotextured layer stacks on thick glass substrates it is imperative to take the air-glass interface into account adequately. Often, one only corrects for initial reflection losses at the air-glass interface, which are about $4 \%$ at normal incidence. We show that it is also very important to take the first-order reflections at the glass-air interface into account, which occurs for light that is reflected from the nanotextured layer stack into glass. The first-order correction leads to much better agreement between measured and simulated reflection data, because reflection orders that are present in glass at large angles cannot propagate into air.

In a next step, we will implement the calculation of the scattering matrix, which connects all the incoming directions to all outgoing directions. Once this scattering matrix is calculated using FEM, an almost arbitrary number of reflection corrections can be evaluated at very little computational cost.

Even though the first-order correction might not be as accurate as the approach, where a large number of correction orders can be determined using the scattering matrix, it is very simple and can be quickly applied to many problems, where a thick glass superstrate cannot be taken into account directly.

\section{ACKNOWLEDGMENTS}

We would like to thank Bernd Rech for the stimulating discussions. We acknowledge the German Federal Ministry of Education and Research (BMBF) for funding via Nachwuchswettbewerb NanoMatFutur (No. 03X5520) and the Einstein Foundation Berlin (ECMath project SE6). The results were obtained at the Berlin Joint Lab for Optical Simulations for Energy Research (BerOSE) of Helmholtz-Zentrum Berlin für Materialien und Energie, Zuse Institute Berlin and Freie Universität Berlin.

\section{REFERENCES}

1. G. Köppel, B. Rech, and C. Becker, "Sinusoidal nanotextures for light management in silicon thin-film solar cells," Nanoscale 8, pp. 8722-8728, 2016.

2. K. Jäger, C. Barth, M. Hammerschmidt, S. Herrmann, S. Burger, F. Schmidt, and C. Becker, "Simulations of sinusoidal nanotextures for coupling light into c-Si thin-film solar cells," Opt. Express 24, pp. A569-A580, 2016.

3. D. Eisenhauer, G. Köppel, K. Jäger, D. Chen, O. Shargaieva, P. Sonntag, D. Amkreutz, B. Rech, and C. Becker, "Smooth anti-reflective three-dimensional textures for liquid phase crystallized silicon thin-film solar cells on glass," Sci. Rep. 7, p. 2658, June 2017.

4. J. Haschke, D. Amkreutz, and B. Rech, "Liquid phase crystallized silicon on glass: Technology, material quality and back contacted heterojunction solar cells," Jpn. J. Appl. Phys. 55(4S), p. 04EA04, 2016.

5. P. Sonntag, N. Preissler, M. Bokalič, M. Trahms, J. Haschke, R. Schlatmann, M. Topič, B. Rech, and D. Amkreutz, "Silicon Solar Cells on Glass with Power Conversion Efficiency above $13 \%$ at Thickness below 15 Micrometer," Sci. Rep. 7, p. 873, Apr. 2017.

6. V. Preidel, D. Amkreutz, J. Haschke, M. Wollgarten, B. Rech, and C. Becker, "Balance of optical, structural, and electrical properties of textured liquid phase crystallized Si solar cells," J. Appl. Phys. 117, p. 225306, 2015.

7. G. Köppel, D. Eisenhauer, B. Rech, and C. Becker, "Combining tailor-made textures for light in-coupling and light trapping in liquid phase crystallized silicon thin-film solar cells," Opt. Express 25, pp. A467-A472, Jun 2017.

8. C. Battaglia, J. Escarré, K. Söderström, M. Charrière, M. Despeisse, F. Haug, and C. Ballif, "Nanomoulding of transparent zinc oxide electrodes for efficient light trapping in solar cells," Nat. Photonics 5, p. 535, 2011. 
9. A. Mellor, H. Hauser, C. Wellens, J. Benick, J. Eisenlohr, M. Peters, A. Guttowski, I. Tobías, A. Martí, A. Luque, and B. Bläsi, "Nanoimprinted diffraction gratings for crystalline silicon solar cells: implementation, characterization and simulation," Opt. Express 21, pp. A295-A304, Mar 2013.

10. M. Verschuuren and H. van Sprang, "3D Photonic Structures by Sol-Gel Imprint Lithography," Mater. Res. Soc. Symp. Proc. 1002, pp. 1002-N03-05, 2007.

11. J. Jin, The Finite Element Method in Electromagnetics, John Wiley \& Sons, 2002.

12. A. Schädle, L. Zschiedrich, S. Burger, R. Klose, and F. Schmidt, "Domain decomposition method for Maxwell's equations: Scattering off periodic structures," J. Comput. Phys. 226(1), pp. 477 - 493, 2007.

13. M. A. Green, A. Ho-Baillie, and H. J. Snaith, "The emergence of perovskite solar cells," Nat. Photon. 8, pp. 506-514, July 2014.

14. F.-J. Haug and C. Ballif, "Light management in thin film silicon solar cells," Energy Environ. Sci. 8, pp. 824-837, 2015.

15. O. Isabella, S. Solntsev, D. Caratelli, and M. Zeman, "3-D optical modeling of thin-film silicon solar cells on diffraction gratings," Prog. Photovolt: Res. Appl. 21, pp. 94-108, 2013.

16. K. Jäger, G. Köppel, C. Barth, M. Hammerschmidt, S. Herrmann, S. Burger, F. Schmidt, and C. Becker, "Sinusoidal gratings for optimized light management in c-Si thin-film solar cells," Proc. SPIE 9898, p. 989808, 2016.

17. J. Pomplun, S. Burger, L. Zschiedrich, and F. Schmidt, "Adaptive finite element method for simulation of optical nano structures," Phys. Status Solidi B 244, pp. 3419-3434, 2007. http://jcmwave.com. 